\title{
Four-qubit Systems and Dyonic Black Hole-Black Branes in Superstring Theory
}

\author{
A. Belhaj ${ }^{1,2}$, M. Bensed ${ }^{3}$, Z. Benslimane ${ }^{3}$, M. B. Sedra ${ }^{3}$, A. Segui $^{2}$ \\ ${ }^{1}$ LIRST, Département de Physique, Faculté Polydisciplinaire, Université Sultan Moulay Slimane \\ Béni Mellal, Morocco \\ ${ }^{2}$ Departamento de Física Téorica, Universidad de Zaragoza, E-50009-Zaragoza, Spain \\ ${ }^{3}$ Département de Physique, LabSIMO, Faculté des Sciences, Université Ibn Tofail
}

Kénitra, Morocco

\begin{abstract}
Using dyonic solutions in the type IIA superstring theory on Calabi-Yau manifolds, we reconsider the study of black objects and quantum information theory using string/string duality in six dimensions. Concretely, we relate four-qubits with a stringy quaternionic moduli space of type IIA compactification associated with a dyonic black solution formed by black holes $(\mathrm{BH})$ and black 2-branes (B2B) carrying 8 electric charges and 8 magnetic charges. This connection is made by associating the cohomology classes of the heterotic superstring on $T^{4}$ to four-qubit states. These states are interpreted in terms of such dyonic charges resulting from the quaternionic symmetric space $\frac{S O(4,4)}{S O(4) \times S O(4)}$ corresponding to a $N=4$ sigma model superpotential in two dimensions. The superpotential is considered as a functional depending on four quaternionic fields mapped to a class of Clifford algebras denoted as $C l_{0,4}$. A link between such an algebra and the cohomology classes of $T^{4}$ in heterotic superstring theory is also given.
\end{abstract}

Keywords: Qubit information systems; superstring theory; string/string duality; quaternionic manifolds. 


\section{Introduction}

Extremal black branes have been extensively studied in the framework of superstring theory on the Calabi-Yau (CY) manifolds [1, 2, 3, 4]. These black solutions have been approached by exploring the attractor mechanism and the topological string theory [5, 6, 7, 8, 9]. In the attractor mechanism scenario, the scalars could be fixed in terms of the black brane charges by extremising the associated potential with respect to the stringy moduli obtained from the superstring theory compactified on the Calabi-Yau manifolds. Moreover, the corresponding entropy functions have been computed using the string duality symmetries acting on the invariant black brane charges. In this way, several Calabi-Yau compactifications have been examined producing various results dealing with black objects in type II superstrings using D-brane physics [10, 11].

The black objects, embedded in superstring theory compactifications, can be connected to quantum information theory using the qubit analysis [12-24]. Precisely, a fascinating correspondence has been discovered between quantum information theory and superstring theory. The main obtained relations are between the entropy formulas for specific black hole solutions in supergravity theories and entanglement measures for certain multiqubit systems [17, 18, Alternative studies have been conducted using toric geometry and graph theory [24, 25, 26, 27]. The underlying idea is a link between the $N=2$ STU black hole charges and three-qubit states which has been established in [15, 16]. Furthermore, the analysis based on three-qubits has been developed to describe the structure of extremal black hole solutions in terms of four-qubit systems [12, 19, 22, 23, 28]. In all the works on four-qubits, one uses the complex geometry to deal with the corresponding black hole entropy. For more details, we refer to [22, 29].

The main goal of this work is to contribute to these activities by approaching four-qubit systems using string dualities and a quaternionic description of stringy moduli spaces. Concretely, we reconsider the investigation of black objects and quantum information theory using string/string duality in the context of dyonic solutions in type II superstring compactifications on Calabi-Yau manifolds. This may offer a new take on the moduli space of black objects and quantum information theory. More precisely, we link four-qubits with a stringy quaternionic moduli space of type IIA superstring compactification associated with a dyonic black solution formed by black holes (BH) and back 2-branes (B2B), referred to as $\left(\begin{array}{l}0 \\ 2\end{array}\right)$ dyonic object with eight electric charges and eight magnetic charges, producing sixteen charges. This connection is made by associating the cohomology classes of the heterotic superstring on $T^{4}$ to four-qubit states. These states can be interpreted in terms of charges of such dyonic solutions resulting from the quaternionic symmetric space $\frac{S O(4,4)}{S O(4) \times S O(4)}$ corresponding to a superpotential of $N=4$ sigma model in two dimensions. The superpotential has been considered as a functional de- 
pending on quaternionic fields related to a class of the Clifford algebras developed in [30]. This algebra, denoted as $C l_{0,4}$, provides a link with the cohomology classes of $T^{4}$ in the heterotic superstring compactification.

The organization of the paper is as follows. Section 2 is a concise review on the study of dyonic solutions in type II superstrings compactified on $n$-dimensional Calabi-Yau manifolds. We emphasize the black solutions carrying charges in $10-2 n$ dimensions sharing electric and magnetic charge dualities. A classification according to black brane dimensions results in two dyonic solutions which can be generalized in arbitrary dimension. Section 3 contains a link between four-qubit systems and a quaternionic geometry considered as a reduction of the moduli space of the heterotic superstring compactified on $T^{4}$ being dual to type IIA superstring on the K3 surface. This moduli space will be considered as the moduli space of a dyonic BHB2B object with 8 electric charges and 8 magnetic charges. In section 4 , the four-qubit states

are related to dyonic charges living in the moduli space $\frac{S O(4,4)}{S O(4) \times S O(4)}$. In section 5 , we claim that the usual decomposition of $S O(4) \times S O(4) \longrightarrow S U(2) \times S U(2) \times S U(2) \times S U(2)$ results in four quaternionic fields that can be interpreted in terms of four-qubit states. We suggest that these fields can produce a quaternionic superpotential associated with a $N=4$ sigma model in two dimensions [31]. Precisely, we regard the superpotentiel as an element of a particular class of the Clifford algebras denoted as $C l_{0,4}$. We end in Section 6 with some discussions and open questions.

\section{Dyonic solutions in type II superstrings}

We start by reconsidering the study of dyonic solutions in type II superstrings compactified on CY manifolds, that we will later need. It is recalled that a $n$-dimensional CY manifold (CY $n$-folds) is a complex with the Kähler structure. The latter involves a global nonvanishing holomorphic $n$-form which is equivalently to a Kähler manifold with a vanishing first Chern class $c_{1}=0$ required by a $S U(n)$ holonolmy group [32]. The superstring compactification on such manifolds preserves only $\frac{1}{2^{n-1}}$ of the ten dimensional supercharges. It has been remarked that each manifold is associated with a Hodge diagram playing an important rôle in the determination of the superstring theory spectrum in $10-2 n$ dimensions [32, 33, 34, 35]. To have a general idea on such data, we list the Hodge diagrams of the $T^{2}$ torus, the K3 surface and the 
CY threefolds, respectively:

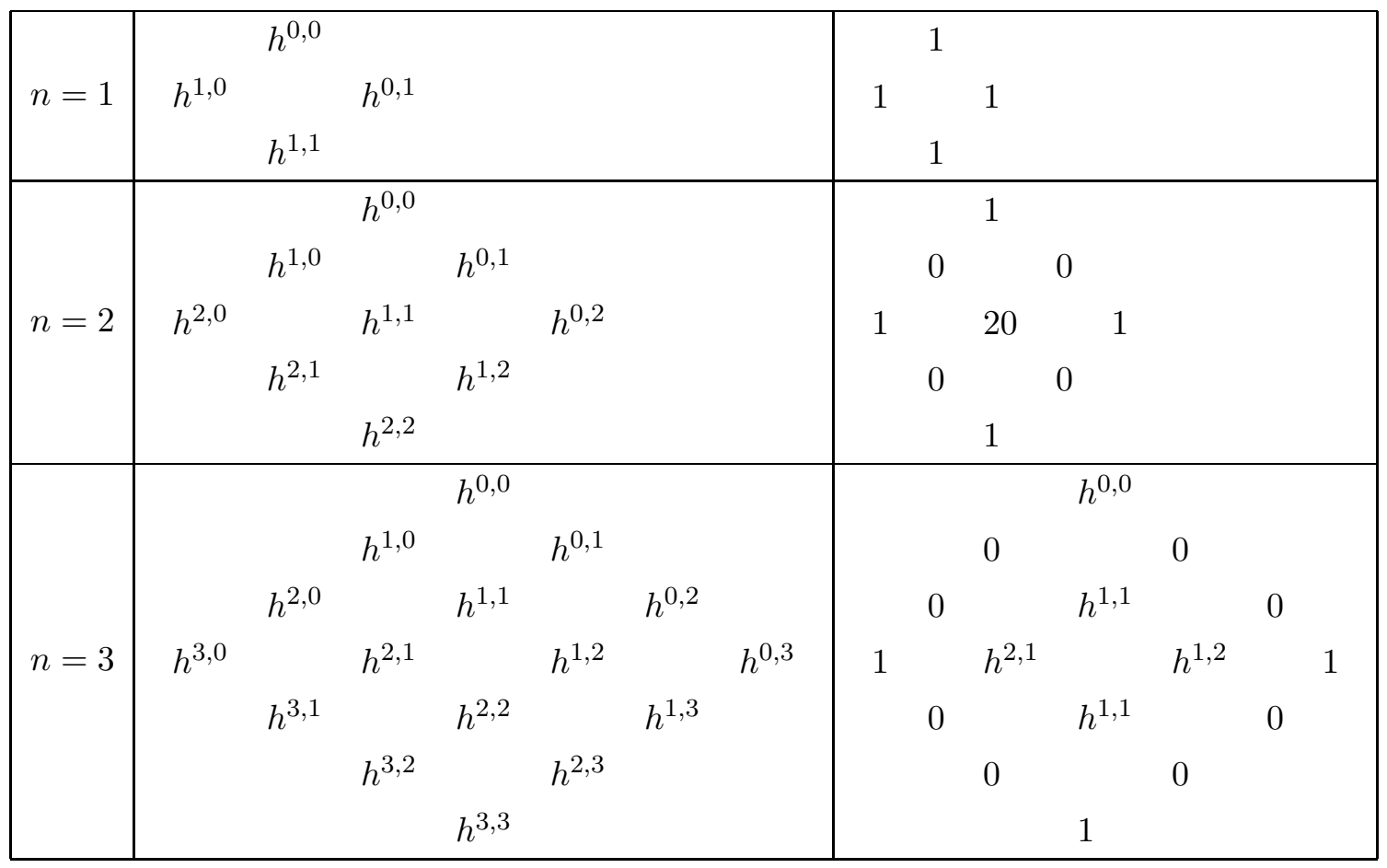

It turns out that $h^{p, q}$ denotes the number of the holomorphic and the anti holomorphic $(p, q)$ forms. Deleting the zeros, one observes that each diagram contains two central orthogonal lines. For the CY $n$-folds, the vertical line encodes the parameters describing the Kähler deformations. It has been shown that the number of such size parameters, representing the Kähler deformations of the metric, is fixed by $h^{1,1}$. The horizontal one represents the parameters of the complex structure (shape parameters) given by $h^{n-1,1}$. Beside these parameters, the Hodge diagram can be explored to produce the all physical data in lower dimensional superstring theory compactifications. Indeed, the moduli space of the CY type II superstring compactifications is determined by the geometric deformations of the CY metric including the antisymmetric B-field of the NS-NS sector, the dilaton, specifying the string coupling constant and the scalars derived from the $\mathbf{R}-\mathbf{R}$ gauge fields on non trivial cycles of the CY spaces. In connection with the black solutions in the type II superstring compactifications, these scalar fields which are associated with supergravity models having $2^{6-n}$ supercharges are coupled to an abelian gauge symmetry providing electric and magnetic charges of black objects in $10-2 n$ dimensions. In this way, the near horizon of these black objects is usually defined by the product of Ads spaces and spheres as follows

$$
A d s_{p+2} \times S^{8-2 n-p}
$$

where $p$ is the internal dimension of the black brane. $n$ and $p$ verify the following constraint

$$
2 \leq 8-2 n-p
$$


In the compactified theory living in $10-2 n$, the electric/magnetic duality liking a $p$-dimensional electrical black brane to a $q$-dimensional magnetic one is assured by the constraint

$$
p+q=6-2 n
$$

A priori, this equation can be solved in different ways according to the $(p, q)$ couple values. The solution can be classified as follows

- $(p, q)=(0,6-2 n)$ describing an electrical charged black hole $(\mathrm{BH})$

- $(p, q)=(3-n, 3-n)$ describing dyonic black branes $(\mathrm{DB}(3-n) \mathrm{B})$

- $(p, q) \neq(0,6-2 n)$ and $(p, q) \neq(3-n, 3-n)$, describing black objects like strings, membranes and higher-dimensional branes.

However, a closed inspection shows that we have two kinds of dyonic solutions carrying electric and magnetic charges by considering objet like doublets. They are listed as follows

1. a solution $\left(\begin{array}{c}3-n \\ 3-n\end{array}\right)$ consisting of the same object associated with

$$
p=q=3-n .
$$

2. a solution $\left(\begin{array}{c}p \\ 6-2 n-p\end{array}\right)$ consisting of an electrically charged black object and its magnetic dual one corresponding to

$$
p, \quad q=6-2 n-p, \quad p \neq 3-n .
$$

The last solution is considered as a single object sharing similar features of the usual dyonic solution described by the same object. We believe that one could build it in any dimension. To see that, let us consider a model obtained by the compactification of type IIA superstring on the K3 surface required by the $h^{1,0}=h^{1,2}=h^{0,1}=h^{2,1}=0$. Indeed, it is recalled that the type IIA superstring perturbative bosonic massless sector contain the following fields

$$
\text { NS-NS : } g_{M N}, B_{M N}, \phi \quad \mathbf{R}-\mathbf{R}: A_{M}, C_{M N K}
$$

where $M, N, K=0, \ldots, 9$. This compactification produces a $N=2$ supergravity in six dimensions with the following bosonic spectrum

$$
g_{\mu \nu}, B_{\mu \nu}, \phi, A_{\mu}, C_{\mu \nu \rho}, C_{\mu i j}, \quad \phi_{\alpha}, \alpha=1, \ldots, 80 .
$$

In this spectrum, $g_{\mu \nu}$ is the six dimensional graviton metric, $B_{\mu \nu}$ and $C_{\mu \nu \rho}$ are the six dimensional antisymmetric gauge fields. The field $A_{\mu}$ and $C_{\mu i j}$ represent the gravi-photon and 
Maxwell gauge fields in six dimensions. These fields are obtained from the compactification of $C_{\mu \nu \rho}$ on the real 2-cycles of the K3 surface. Since $C_{\mu \nu \rho}$ is dual to a vector in six dimensions, the theory has an $U(1)^{24}$ abelian gauge symmetry. Besides the antisymmetric gauge field $B_{\mu \nu}$, the total gauge symmetry reads

$$
G=G_{1} \times G_{2}=U(1)^{24} \times U(1)
$$

associated with one and two-form gauge fields, respectively. In six dimensions, these gauge fields are coupled to the scalar fields. In addition to the dilaton $\phi$ in six dimensions, there are 80 scalar fields $\phi_{\alpha}$ which can be arranged to form the moduli space of type IIA superstring on the K3 surface. The latter can be viewed as a scalar manifold of half-maximal, non-chiral Type IIA supergravity in six dimensions, coupled to 20 vector multiplets, which reads as

$$
\frac{S O(4,20)}{S O(4) \times S O(20)} \times S O(1,1)
$$

It has been shown that the first factor $\frac{S O(4,20)}{S O(4) \times S O(20)}$ represents the geometric deformations of the K3 surface in the presence of the antisymmetric B-field of the NS-NS sector and it is linked to the symmetry group $G_{1}=U(1)^{24}$. However, the second factor $S O(1,1)$ represents the dilaton scalar field which is associated with $G_{2}=U(1)$ [35, 36]. It has been remarked that the space (2.9) is related to the electric/magnetic duality assured by the condition

$$
p+q=2
$$

producing two possible dynonic solutions
1. $\left(\begin{array}{l}0 \\ 2\end{array}\right)$ BH-B2B with near-horizon geometries $A d s_{2} \times S^{4}$ and $A d s_{4} \times S^{2}$
2. $\left(\begin{array}{l}1 \\ 1\end{array}\right)$ black string $(\mathrm{BS})$ with the near horizon geometry $A d s_{3} \times S^{3}$.

In six dimensions, the factor $\frac{S O(4,20)}{S O(4) \times S O(20)}$ corresponds to the dyonic $\mathrm{BH}-\mathrm{B} 2 \mathrm{~B}$ object $\left(\begin{array}{l}0 \\ 2\end{array}\right)$ with 24 electric charges and 24 magnetic charges. It can be realized in terms of a D-brane system containing $\{D 0, D 2, D 4, D 6\}$ which can be placed on the corresponding Hodge diagram. The $24+24$ charges are represented by the configuration of the K3 surface as follows

$(D 0, D 2)$

$1201 \equiv(D 2, D 4) \quad(D 2, D 4) \quad(D 2, D 4)$ 
However, the factor $S O(1,1)$ is associated with the dyonic BS $\left(\begin{array}{l}1 \\ 1\end{array}\right)$ having one electric charge and one magnetic charge. It is represented by the following D-brane configuration on the K3 surface

$$
\begin{array}{ccccc} 
& & & F 1 \\
1 & 20 & 1 & & \\
& & & \\
1 & & N S 5
\end{array} .
$$

Having discussed the compactification of the type IIA superstring on the K3 surface, we would like to relate the corresponding black objects and quantum information theory by combining string/string duality and non trivial cycles appearing in the toroidal compctification of the heterotic superstring on $T^{4}$. A special emphasis is put on four-qubit systems which will be linked with a particular quaternionic geometry considered as a subspace of the one appearing in (2.9).

\section{Four-qubit systems and dyonic solutions on the symmetric space $\frac{S O(4,4)}{S O(4) \times S O(4)}$}

Several structural similarities between quantum information theory and superstring theory have been established forming the so called black hole qubit correspondence (BHQC). The first mapping was between the entropy formulae of certain black holes and the entanglement measures of qubit systems using Cayley's hyperdeterminant [37, 38, 39]. In particular, it has been shown that the square root of Cayley's hypertederminant is linked to eight charges of extremal black holes in the STU model by the entropy formulae as follow

$$
S=\pi \sqrt{\left|\operatorname{Deta}_{A B C}\right|}=\frac{\pi}{2} \sqrt{\tau_{A B C}}
$$

Here $\tau_{A B C}$ and $S$ are the 3 -tangle measure and the black hole entropy, respectively. Furthermore, it is clearly interesting to recall that the Cayley's hyperderminant denoted as $\operatorname{Det} A$ is defined as

$$
\operatorname{Det} A \equiv-\frac{1}{2} \epsilon^{A_{1} A_{3}} \epsilon^{A_{2} A_{4}} \epsilon^{B_{1} B_{2}} \epsilon^{B_{3} B_{4}} \epsilon^{C_{1} C_{2}} \epsilon^{C_{3} C_{4}} a_{A_{1} B_{1} C_{1}} a_{A_{2} B_{2} C_{2}} a_{A_{3} B_{3} C_{3}} a_{A_{4} B_{4} C_{4}} .
$$

It is not hard to see that this is an homogeneous quartic polynomial that involves an interesting physical interpretation in terms of the STU black hole charges embedded in type II superstrings. Precisely, the solution of the STU black hole, in the case of spherical symmetry, is given in terms of 8 charges $\left(q_{0}, q_{1}, q_{2}, q_{3}, p^{0}, p^{1}, p^{2}, p^{3}\right)$. In this way, the square of the extremal STU black 
hole entropy is proportional to a quartic polynomial of $q_{0}, q_{1}, q_{2}, q_{3}, p^{0}, p^{1}, p^{2}$ and $p^{3}$ [15, 16]

$$
\begin{aligned}
S^{2}= & \pi^{2}\left\{-\left(p^{0} q_{0}+p^{1} q_{1}+p^{2} q_{2}+p^{3} q_{3}\right)^{2}\right. \\
& +4\left(\left(p^{1} q_{1}\right)\left(p^{2} q_{2}\right)+\left(p^{1} q_{1}\right)\left(p^{3} q_{3}\right)+\left(p^{3} q_{3}\right)\left(p^{2} q_{2}\right)\right. \\
& \left.\left.+q_{0} p^{1} p^{2} p^{3}-p^{0} q_{1} q_{2} q_{3}\right)\right\} .
\end{aligned}
$$

Under a suitable mapping, the eight charges of the STU black hole correspond to the states of a three-qubit system as follows [4]

$$
\left(\begin{array}{c}
q_{0} \\
q_{1} \\
q_{2} \\
q_{3} \\
p^{0} \\
p^{1} \\
p^{2} \\
p^{3}
\end{array}\right) \quad \longleftrightarrow \quad\left(\begin{array}{c}
a_{000} \\
-a_{001} \\
-a_{010} \\
-a_{100} \\
a_{111} \\
a_{110} \\
a_{101} \\
a_{011}
\end{array}\right)
$$

Motivated by the STU black hole, three-qubits and certain extended works, we would like to link the four-qubits with a particular stringy moduli space given by

$$
\frac{S O(4,4)}{S O(4) \times S O(4)}
$$

We will show that this moduli space can be considered as a subpart of a general quaternionic geometry, related to the c-map image of the symmetric projective special Khler manifold $S L(2, R)^{3} / U(1)^{3}$, which is the vector multiplets scalar manifold of the $N=2 D=4 \mathrm{STU}$ supergravity model [48]. Then, we will show that this moduli space corresponds to a dyonic black object in six dimensions carrying eight electric and eight magnetic charges playing the same role as a black string in six dimensions. Concretely, these black solutions are mapped to four-qubit physical systems using string dualities. It is recalled that the physics of the qubit has been extensively investigated from different physical and mathematical aspects [40, 41, 42. Using Dirac notation, one-qubit is described by the following state

$$
|\psi\rangle=a_{0}|0\rangle+a_{1}|1\rangle
$$

Here, $a_{i}$ are considered as complex numbers verifying the probability condition

$$
\left|a_{0}\right|^{2}+\left|a_{1}\right|^{2}=1
$$

It should be denoted that this condition can be interpreted geometrically in terms of the socalled Bloch sphere, $\mathbb{C P}^{1}$. Similarly, the two-qubits are represented by the general state

$$
|\psi\rangle=a_{00}|00\rangle+a_{10}|10\rangle+a_{01}|01\rangle+a_{11}|11\rangle
$$


In this case, the probability condition is

$$
\left|a_{00}\right|^{2}+\left|a_{10}\right|^{2}+\left|a_{01}\right|^{2}+\left|a_{11}\right|^{2}=1
$$

defining a 3-dimensional complex projective space $\mathbb{C P}^{3}$ generalizing the Bloch sphere. This analysis can be extended to $N$-qubits having $2^{N}$ configuration states. For instance, the general state of the four-qubits reads as

$$
|\psi\rangle=\sum_{i j k \ell=0,1} a_{i j k \ell}|i j k \ell\rangle
$$

where $a_{i j k \ell}$ verify the normalization condition

$$
\sum_{i j k \ell=0,1} a_{i j k \ell} \bar{a}_{a_{i j k \ell}}=1
$$

defining the $\mathbb{C P}^{15}$ complex projective space. Using results on string dualities, these states will be linked to charges of a dyonic object embedded in type IIA superstring.

Roughly speaking, the moduli space (3.4) can be considered as a particular geometry of

$$
\frac{S O(4, m)}{S O(4) \times S O(m)} \times S O(1,1)
$$

where $m \geq 3$ is an integer which can be fixed by the compactification in question. It appears naturally in six dimensional supergravity models.

The link that we are after push us to consider the case $m=4$ reducing the above moduli space to

$$
\frac{S O(4,4)}{S O(4) \times S O(4)} \times S O(1,1)
$$

It turns out that in the analysis of the superstring compactifications (sigma model fields), we can remove the factor $S O(1,1)$ by fixing the dilaton. The remaining factor can be obtained using different ways. A possible one is to think about the decomposition of the moduli space of the K3 surface, or the heterotic superstring on $T^{4}$. It has been shown that such two models are equivalent. This duality is known by string/string duality in six dimensions [43]. Indeed, it is possible to use the following decomposition

$$
\frac{S O(4,20)}{S O(4) \times S O(20)} \rightarrow \frac{S O(4,4)}{S O(4) \times S O(4)} \times \frac{S O(4,16)}{S O(4) \times S O(16)}
$$

supported by the fact that

$$
4 \times 20=4 \times 4+4 \times 16 .
$$

Examining the string/string duality in six dimensions, the factor $\frac{S O(4,16)}{S O(4) \times S O(16)}$ corresponds to the twistor sector in type IIA superstring side. It is associated with the fixed points of the orbifold compactification. It is interesting to note that this sector has played a primordial 
rôle in solving a serious problem in type IIA spectrum in six dimensions being the absence of non abelian gauge symmetries. However, this sector will be ignored and we consider only the factor $\frac{S O(4,4)}{S O(4) \times S O(4)}$. This will be done by restricting quaternionic dimensions living in six dimensional supergravity where certain parts of stringy moduli space should take zero values. In superstring theory, this factor can be obtained from the toroidoal compactification of the heterotic superstring by ignoring the contribution of the gauge symmetry derived from the 26-dimensional bosonic sector. In particular, the compactification of the heterotic superstring on $T^{4}$ produces $\frac{4 \times 5}{2}$ degrees of freedom associated with the metric $g_{i j}$ and $\frac{4 \times 3}{2}$ degrees of freedom corresponding to the anti-symmetric field $B_{i j}$. Then, we have $4 \times 4=16$ real scalars parameterizing the symmetric space $\frac{S O(4,4)}{S O(4) \times S O(4)}$. Besides such scalar fields, we have also the configuration representing the abelian gauge fields. These abelian gauge fields can be obtained from the $B_{\mu i}$ and $g_{\mu i}$ fields. This generates the gauge symmetry

$$
G_{1}=U(1)^{4} \times U(1)^{4}
$$

which provides a $S O(4) \times S O(4)$ isotopy symmetry. In six dimensions, this symmetry corresponds to

- 8 electric charges of $\mathrm{BH}$ solution with the $A d S_{2} \times S^{4}$ near horizon geometry.

- 8 magnetic charges of a B2B solution with the $A d S_{4} \times S^{2}$ near horizon geometry.

Following the general discussion made in the previous section, the moduli space $\frac{S O(4,4)}{S O(4) \times S O(4)}$ corresponds to a BH-B2B dyonic object $\left(\begin{array}{l}0 \\ 2\end{array}\right)$ with 8 electric charges and 8 magnetic charges. It can be realized in terms of a D-brane system containing $\{D 0, D 2, D 4, D 6\}$ which can be placed on the Hodge diagram of $T^{4}$. At the level, it is intersecting to note that this solution could generate a single dyonic object in four dimensions. Assuming that the corresponding gauge fields survive in four dimensions, the $\mathrm{B} 2 \mathrm{~B}$ can be converted to a $\mathrm{BH}$ in four dimensions using a possible compactification on a 2 -sphere $S^{2}$

$$
B 2 B \stackrel{S^{2}}{\longrightarrow} B H
$$

This could produce a single dyonic solution $\left(\begin{array}{l}0 \\ 0\end{array}\right)$ in four dimensions with the near-horizon geometry $A d S_{2} \times S^{2}$ by thinking $A d S_{4} \times S^{2}$ as $A d S_{2} \times S^{2} \times S^{2}$. In what follows, the dyonic black solution $\left(\begin{array}{l}0 \\ 2\end{array}\right)$ can be considered as a four-qubit system supported by string/string duality in six dimensions [43]. 


\begin{tabular}{|c|c|}
\hline (p,q)forms & number \\
\hline \hline 1 & 1 \\
\hline$d z_{1}, \overline{d z_{1}}, d z_{2}, \overline{d z_{2}}$ & 4 \\
\hline$d z_{1} \wedge \overline{d z}_{1}, d z_{1} \wedge d z_{2}, d z_{1} \wedge \overline{d z}_{1}$ & 3 \\
\hline$d z_{2} \wedge \overline{d z}_{2}, d z_{2} \wedge d z_{1}, d z_{2} \wedge \overline{d z}_{1}$ & 3 \\
\hline$d z_{1} \wedge \overline{d z}_{1} \wedge \overline{d z}_{2}, d z_{1} \wedge \overline{d z}_{1} \wedge d z_{2}, \overline{d z_{1}} \wedge d z_{2} \wedge \overline{d z}_{2}, d z_{1} \wedge d z_{2} \wedge \overline{d z}_{2}$ & 4 \\
\hline$d z_{1} \wedge \overline{d z}_{1} \wedge d z_{2} \wedge \overline{d z}_{2}$ & 1 \\
\hline
\end{tabular}

Table 1: $(p, q)$-forms on $T^{2} \times T^{2}$.

\section{String/string duality interpretation of four-qubits}

In this section, we would like to present a stringy interpretation of four-qubits using the string/string duality relating type IIA and heterotic superstings [43]. Indeed, instead of thinking in terms of type IIA D-barnes wrapping non trivial cycles, as done in the second section of the present work, we consider an equivalent description in heterotic superstring using cycles in $T^{4}$. More precisely, we associate to each element of the cohomology classes of the heterotic superstring on $T^{4}$ a state of the four-qubit basis. The basis states can be interpreted in terms of the trivial fibration $T^{4}=T^{2} \times T^{2}$. To see that, let us consider the complex realization of $T^{2} \times T^{2}$,

$$
\begin{aligned}
& z_{\alpha}=z_{\alpha}+1 \\
& z_{\alpha}=z_{\alpha}+i, \quad i^{2}=-1, \quad \alpha=1,2 .
\end{aligned}
$$

The cohomology classes of this trivial fibration correspond to the holomorphic and the antiholomorphic forms $(p, q)$ which are listed in table 1 .

The table arrangement is motivated from the $2^{4}=C_{4}^{0}+C_{4}^{1}+C_{4}^{2}+C_{4}^{3}+C_{4}^{4}$ which can be explored to divide the 2 -forms into two categories

$$
\begin{aligned}
& d z_{1} \wedge \overline{d z}_{1}, d z_{1} \wedge d z_{2}, d z_{1} \wedge \overline{d z}_{2} \\
& d z_{2} \wedge \overline{d z_{2}}, \overline{d z}_{1} \wedge d z_{2}, \overline{d z}_{1} \wedge \overline{d z_{2}}
\end{aligned}
$$

as required by the normalized volume form on $T^{2} \times T^{2}$

$$
\int_{T^{4}} d z_{1} \wedge \overline{d z}_{1} \wedge d z_{1} \wedge \overline{d z}_{2}=1 .
$$

To make contact with four-qubit states, we consider the following map applied first on one factor $T^{2}$

$$
\omega_{i j}^{1}=\left(d z_{1}\right)^{i} \wedge\left(\overline{d z_{1}}\right)^{j} \longrightarrow \mid i j>i, j=0,1
$$


producing the two-qubit states. Similarly, the basis states of four-qubits can be obtained by fibering trivially the $T^{2} \times T^{2}$ complex manifold. Indeed, we define the factorization

$$
\omega_{i j k \ell}=\omega_{i j}^{1} \wedge \omega_{k \ell}^{2}=\left(d z_{1}\right)^{i} \wedge\left(\overline{d z_{1}}\right)^{j} \wedge\left(d z_{2}\right)^{k} \wedge\left(\overline{d z_{2}}\right)^{\ell}, \quad i, j, k, \ell=0,1
$$

representing the basis states of the four-qubits

$$
\omega_{i j k l} \longrightarrow \mid i j k \ell>
$$

The normalized condition may be assured by

$$
\left(\omega_{i j k \ell}, \omega_{i^{\prime} j^{\prime} k^{\prime} \ell^{\prime}}\right)=\delta_{i}^{i^{\prime}} \delta_{j}^{j^{\prime}} \delta_{k}^{k^{\prime}} \delta_{\ell}^{\ell^{\prime}}
$$

where the scalar product can be defined by

$$
\left(\omega_{i j i^{\prime} j^{\prime}}, \omega_{k l m n}\right)=\int_{T^{2}} \omega_{i j i^{\prime} j^{\prime}} \wedge * \omega_{k l m n} .
$$

Here $*$ is the Hodge duality. In order to establish a connection with the dyonic solutions in type IIA supersstring, each state $\mid i j k \ell>$ should correspond to a charge of the following D-brane system

$$
\{D 0, D 2, D 4, D 6\}
$$

in the presence of $U(1)^{8}$ gauge fields rotated by the $S O(4) \times S O(4)$ isotropy symmetry. In the heterotic superstring side, these vectors, obtained from the graviton and the antisymmetric B-field, can be split as

$$
8=1+3+4
$$

This decomposition can be supported by the fact that a real vector of $S O(4)$ splits under the $\frac{1}{2} \times \frac{1}{2}$ spin representation of $S U(2)$ as a 1-singlet and a triplet as follows

$$
4=1+3
$$

This matches perfectly with the above table of the differential complex forms arrangement on the trivial fibration of $T^{2} \times T^{2}$. In this way, the eight electric charges are linked with $1+3$ vectors of type $g_{\mu a}$ and 4 vectors of type $B_{\mu a}$ of the heterotic superstring in six dimensions. The 8 magnetic charges $q_{a}$ can be associated with the dual objects as required by the electric and magnetic duality

$$
p_{a} q^{a}=2 \pi k
$$

These objects forming a dyonic pair of a black solution $\left(\begin{array}{l}0 \\ 2\end{array}\right)$ carrying 8 electric and 8 magnetic charges are associated with the four-qubit states. 


\section{$5 \quad$ Quaternionic description of four-qubits}

The quaternionic character of the moduli space of type IIA superstring on K3 surface, or heterotic superstring on $T^{4}$ pushes us to think about a quaternionic analysis of four-qubits. This could help to clarify ceratin issues by drawing a clear contrast between the attractor mechanism of black holes developed in superstring theory and quantum information theory. In particular, we would like to give such a description using the symmetric space $\frac{S O(4,4)}{S O(4) \times S O(4)}$ parameterized by 16 scalar fields associated with a dyonic solution $\left(\begin{array}{l}0 \\ 2\end{array}\right)$ with 8 electric charges and 8 magnetic charges. It is recalled that a quaternionic field takes the form

$$
q=x_{0}+i x_{1}+j x_{2}+k x_{3},
$$

where $x_{0}, x_{1}, x_{2}$ and $x_{3}$ are real numbers, $i, j$ and $k$ are imaginary numbers such that

$$
\left\{\begin{array}{l}
i^{2}=j^{2}=k^{2}=-1 \\
i j=-j k=k, j k=-k j=1 \quad k i=-k i=j .
\end{array}\right.
$$

Usually, it is convenient to use the matrix representation of quaternionic fields. It is defined by

$$
q=x_{0} \sigma_{0}+i \vec{x} \vec{\sigma}
$$

where $\vec{x}=\left(x_{1}, x_{2}, x_{3}\right)$ and $\vec{\sigma}=\left(\sigma_{1},-\sigma_{2}, \sigma_{3}\right)$ are the usual Pauli matrices and $\sigma_{0}$ is the $2 \times 2$ identity matrix. In this way, a quaternion number is given by

$$
x_{0}+x_{1} i+x_{2} j+x_{3} i j \longrightarrow\left(\begin{array}{cc}
x_{0}+x_{3} & -x_{1}+x_{2} \\
x_{1}+x_{2} & x_{0}-x_{3}
\end{array}\right) .
$$

It turns out that the scalars of the dyonic solutions, studied in the present work, can be combined to form a quaternionic geometry in terms of four quaternionic blocks. To see that, we first recall that these scalar fields belong to the $(4,4)$ bifundamental representation of $S O(4) \times S O(4)$ symmetry

$$
16=(4,4)
$$

In this way, they are specified by two indices $a$ and $b$,

$$
\phi \equiv \phi_{b}^{a}
$$

Then, we consider the following decomposition of $S O(4) \times S O(4)$ symmetry

$$
S O(4) \times S O(4) \longrightarrow S U(2) \times S U(2) \times S U(2) \times S U(2) .
$$

The corresponding representations are given by four integers $\left(m_{1}, m_{2}, m_{3}, m_{4}\right)$, where $m_{s}$ are dimensions of particle state vector spaces. It is recalled that

$$
m_{s}=2 j_{s}+1, \quad s=1,2,3,4
$$


where $j_{s}$ are spin particles. A priori, there are many ways to decompose the bifundamental representation $(4,4)$ in terms of $\left(m_{1}, m_{2}, m_{3}, m_{4}\right)$. A way, which could be related to quaternionic geometry, is

$$
(4,4)=(4,1,1,1) \oplus(1,4,1,1) \oplus(1,1,4,1) \oplus(1,1,1,4) .
$$

This decomposition shows that the symmetric space $\frac{S O(4,4)}{S O(4) \times S O(4)}$ can be parameterized in terms of four quaternionic fields associated with 16 charges of the $\left(\begin{array}{l}0 \\ 2\end{array}\right)$ dyonic black object. In fact, the scalars can be combined to form four quaternionic fields indicated by only one index

$$
\phi_{b}^{a} \longrightarrow \phi_{b_{1} b_{2}}^{a} \longrightarrow \phi_{b_{1} b_{2}}^{a_{1} a_{2}} \longrightarrow \phi^{A}, \quad A=1,2,3,4
$$

where $a_{1}$ and $a_{2}$ refers to the $S U(2)$ group, the same for $b_{1}$ and $b_{2}$. These quaternionic fields can be explored to produce a superpotential of $N=4$ sigma model in two dimensions

$$
W=W\left(\phi^{1}, \phi^{2}, \phi^{3}, \phi^{4}, p^{a}, q^{a}\right) \quad a=1, \ldots, 8 .
$$

In what follows, we will show that this superpotential can be viewed as a general state of fourqubit systems. Indeed, the non commutativity character of the quaternionic fields can be used to make contact with a particular class of the Clifford algebras. In this way, the superpotentiel $W(\phi)$ can be interpreted as an element of such a Clifford algebra. Assuming that the fields $\phi_{A}$ form a normalized basis of a vector space $V$ and using the work developed in [30, the algebra spanned by the all reduced products of the form

$$
\operatorname{Span}\left\{\phi_{1}^{i} \phi_{2}^{j} \phi_{3}^{k} \phi_{4}^{l}\right\}, \quad i, j, k, \ell=0,1
$$

defines a class of the Clifford algebras, denoted as $C l_{0,4}$. It has been shown that this algebra could be decomposed as follows

$$
C l_{0,4}=\oplus_{k=0}^{4} C l_{0,4}^{(k)}
$$

where $C l_{0,4}^{(k)}$ is known by the space of $k$-multivectors [30]. In connection with the heterotic superstring compactification, a close inspection shows that the algebra $C l_{0,4}$ can be associated with the cohomology classes of $T^{4}$. More precisely, we have

$$
\left(\begin{array}{c}
C l_{0,4}^{0}: \text { the scalar } \\
C l_{0,4}^{1}: \text { the } 1 \text {-forms } \\
C l_{0,4}^{2}: \text { the } 2 \text {-forms } \\
C l_{0,4}^{3}: \text { the } 3 \text {-forms } \\
C l_{0,4}^{4}: \text { the volume form }
\end{array}\right) .
$$

Inspired by such a decomposition, we propose the mapping

$$
\mid i j k l>\longrightarrow \phi_{1}^{i} \phi_{2}^{j} \phi_{3}^{k} \phi_{4}^{l}, \quad i, j, k, \ell=0,1 .
$$


In this way, the general state of four-qubits corresponds to a quaternionic superpotential

$$
|\psi\rangle=\sum_{i j k \ell=0,1} a_{i j k \ell}|i j k \ell\rangle \longrightarrow W=W\left(\phi_{1}, \phi_{2}, \phi_{3}, \phi_{4}, p^{a}, q^{a}\right) .
$$

In this mapping, the $a_{i j k \ell}$ numbers should correspond to the $\left(p^{a}, q^{a}\right)$ charges of the dyonic object $\left(\begin{array}{l}0 \\ 2\end{array}\right)$. The superpotential could be viewed as the holomorphic sections of the line bundles on four dimensional quernionic manifolds. We expect that these sections may be encoded in non trivial polytopes going beyond the toric graphs associated with the projective complex geometry used in [44].

\section{Conclusion and open questions}

In this work, we have approached four-qubit systems in the context of type II superstring compactifications using string dualities between type IIA and heterotic superstrings. This connection has been elaborated by giving a classification according to black brane dimensions, which results in two kinds of dyonic solutions generalized in arbitrary dimension where the electric and magnetic charges have been linked formally to Calabi-Yau Hodge numbers. We have shown that the four-qubit systems are related to a stringy moduli space $\frac{S O(4,4)}{S O(4) \times S O(4)}$, which is a reduction of a moduli space of the heterotic superstring $T^{4}$ living in six dimentional supergravity model. Using string/string duality, the four-qubit states are related to a dyonic solution BH-B2B $\left(\begin{array}{l}0 \\ 2\end{array}\right)$ carrying 8 electric and 8 magnetic charges $\left(p_{a}, q_{a}\right)$. Moreover, it has been remarked that the usual decomposition of $S O(4) \times S O(4) \longrightarrow S U(2) \times S U(2) \times S U(2) \times S U(2)$ results in four quaternionic fields that can be interpreted as states of the four-qubits. These states are linked with a quaternionic superpotentiel $W(\phi)$ of $N=4$ sigma interpreted as an element of a particular class of the Clifford algebras denoted as $C l_{0,4}$.

The present work comes up with certain open questions related to quantum information theory. It is recalled that interesting works dealing with four-qubits from algebraic geometry point of view including $\mathrm{ADE}$ singularities have been elaborated in [45, 46]. It would be interesting to see if this has any possible connection with such activities. Moreover, it should be of relevance to approach quantum information concepts using quaternionic geometry associated with Clifford algebras. This includes the study of entanglement and quantum discord. It is clearly interesting to better understand such concepts from geometric methods. We anticipate that many concepts used in quantum information, of four-qubits, could be discussed using quaternionic manifolds. This will be addressed elsewhere.

Acknowledgments: The authors would like to thank M. Asorey for discussions. AB would 
like to thank the Departamento de Física Téorica, Universidad de Zaragoza for very kind hospitality and scientific supports during the realization of a part of this work. He also acknowledges the warm hospitality of Montanez and Naz families durante his travel in Spain and he thanks also Hajja Fatima (his mother) for patience and supports. AS is supported by FPA2012-35453.

\section{References}

[1] A. Strominger, C. Vafa, Microscopic Origin of the Bekenstein-Hawking Entropy, Phys.Lett. B379 (1996) 99, arXiv:hep-th/9601029.

[2] C. Vafa, Black Holes and Calabi-Yau Threefolds, Adv.Theor.Math.Phys. 2 (1998) 207, hep-th/9711067.

[3] J. Maldacena, A. Strominger, E. Witten, Black Hole Entropy in M-Theory, JHEP9712 (1997)002, arXiv:hep-th/9711053.

[4] B. Haghighat, S. Murthy, C. Vafa, S. Vandoren, F-Theory, Spinning Black Holes and Multistring Branches, arXiv:1509.00455.

[5] S. Ferrara, R. Kallosh, A. Strominger, $N=2$ Extremal Black Holes, Phys. Rev. D52 (1995) 5412, hep-th/9508072.

[6] S. Ferrara and R. Kallosh, Supersymmetry and Attractors, Phys. Rev. D54 (1996) 1514, hep-th/9602136.

[7] R. Ahl Laamara, M. Asorey, A. Belhaj, A, Segui, Extremal Black Brane Attractors on The Elliptic Curve, J.Phys. A43 (2010) 105401, arXiv:0907.0093.

[8] P. Bueno, R. Davies, C. S. Shahbazi, Quantum black holes in Type-IIA String Theory, arXiv:1210.2817.

[9] H. Ooguri, A. Strominger, C. Vafa, Black Hole Attractors and the Topological String, Phys.Rev. D70(2004)106007, arXiv:hep-th/0405146.

[10] S. Bellucci, S. Ferrara, A. Marrani and A. Yeranyan, Mirror Fermat Calabi-Yau threefolds and Landau-Ginzburg Black Hole Attractors, Riv. Nuov o Cim. 029 (2006)1, hep-th/0608091.

[11] A. Belhaj, On Black Objects in Type IIA Superstring Theory on Calabi-Yau Manifolds, African Journal Of Math. Phys. Vol. 6 (2008)49, arXiv:0809.1114.

[12] M. J. Duff, S. Ferrara, A. Marrani,D = 3 Unification of Curious Supergravities, JHEP

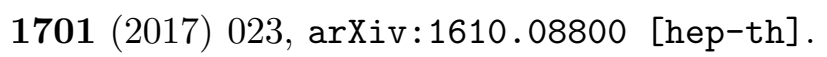


[13] A. Belhaj, M. Bensed, Z. Benslimane, M. B. Sedra, A. Segui, Qubit and Fermionic Fock Spaces from Type II Superstring Black Hole, Int. J. Geom. Methods Mod. Phys. 14 (2017)1750087 arXiv:1604.03998.

[14] A. Belhaj, Z. Benslimane, M. B. Sedra, A. Segui, Qubits from Black Holes in M-theory on K3 Surface, Int. J. Geom. Methods Mod. Phys. 13 (2016)1650075 arXiv:1601.07610.

[15] M. J. Duff, S. Ferrara, Four curious supergravities, Phys. Rev. D83 (2011)046007, arXiv:1010.3173.

[16] P. Levay, Qubits from extra dimensions, Phys. Rev. D84 (2001)125020.

[17] M. J. Duff, String triality, black hole entropy and Cayley s hyperdeterminant, Phys. Rev. D76 (2007) 025017, hep-th/0601134.

[18] P. Levay, Stringy Black Holes and the Geometry of Entanglement, Phys. Rev. D74, 024030 (2006), arXiv:0603136.

[19] P. Levay, F. Holweck, Embedding qubits into fermionic Fock space, peculiarities of the four-qubit case, (2015), arXiv:1502.04537.

[20] P. Levay, F. Holweck, M. Saniga, The magic three-qubit Veldkamp line: A finite geometric underpinning for form theories of gravity and black hole entropy, arXiv:1704.01598.

[21] M. Cvetic, G.W. Gibbons, C.N. Pope, Compactifications of Deformed Conifolds, Branes and the Geometry of Qubits, arXiv:1507.07585.

[22] L. Borsten, D. Dahanayake, M. J. Duff, A. Marrani, W. Rubens, Four-qubit entanglement from string theory, Phys.Rev.Lett. 105 (2010)100507, arXiv:1005.4915,

[23] L. BorstenM. J. DuffA. Marrani,W. Rubens, On the Black-Hole/Qubit Correspondence, Eur. Phys. J. Plus 126 (2011)37, [arXiv:1101.3559 [hep-th].

[24] Y. Aadel, A. Belhaj, M. Bensed, Z. Benslimane, M. B. Sedra, A. Segui, Qubit Systems from Colored Toric Geometry and Hypercube Graph Theory, . Commun. Theor. Phys. 68(2017) 285

[25] A. Belhaj, M. B. Sedra, A. Segui, Graph Theory and Qubit Information Systems of Extremal Black Branes, J.Phys. A48 (2015)045401, arXiv:1406.2578,

[26] A. Belhaj, Multi-qubits and Polyvalent Singularity in Type II Supestring Theory, arXiv:1612.09356. 
[27] A. Belhaj, A. Belhaj, L. Machkouri, M. M. Sedra, S. Ziti, Graph Theory Representation of Quantum Information Inspired by Lie Algebras, arXiv:1609.03534.

[28] P. Levay, STU Black Holes as Four Qubit Systems, Phys. Rev. D82(2010)026003, arXiv:1004.3639.

[29] P. Levay, M. Planat, Metod Saniga, Grassmannian Connection Between Three- and Four-Qubit Observables, Mermin's Contextuality and Black Holes, JHEP 09 (2013)037, arXiv: 1305.5689 .

[30] J. A. Emanuello, Analysis of Functions of Split-Complex, Multicomplex, and SplitQuaternionic Variables and Their Associated Conformal Geometries. PhD thesis, The Florida State University, 2015.

[31] A. Belhaj, Manifolds of $G_{2}$ Holonomy from N=4 Sigma Model, J.Phys. A35(2002)8903, arXiv:hep-th/0201155.

[32] P. Candelas, G. Horowitz, A. Strominger, E. Witten, Vacuum configurations for superstrings, Nucl. Phys. B258 (1985)46.

[33] B.R. Greene, String Theory on Calabi Yau Manifolds, hep-th/9702155.

[34] P. Aspinwall, K3 surfaces and String Duality, hep-th/961117.

[35] A. Belhaj, L.B. Drissi, E.H. Saidi, A. Segui, N=2 Supersymmetric Black Attractors in Six and Seven Dimensions, Nucl. Phys. B796 (2008)521, arXiv:0709.0398.

[36] E.H. Saidi, A. Segui, Entropy of Pairs of Dual Attractors in 6D/7D, JHEP 0807(2008)128, arXiv:0803.2945.

[37] G. Ottavian, Introduction to the Hyperdeterminant and to the Rank of Multidimensional Matrices, arXiv:1301.0472.

[38] A. Cayley, On the theory of linear transformations, Camb. Math. J. 4 193-209,1845.

[39] I.M. Gelfand, M.M. Kapranov, A.V. Zelevinsky Discriminants, Resultants and Multidimensional Determinants, Birkhauser, 1994.

[40] M. A. Nielsen, I. L. Chuang, Quantum Computation and Quantum Information, Cambridge University Press, New York, NY, USA, 2000.

[41] D. R. Terno, Introduction to relativistic quantum information, arXiv:quant-ph/0508049.

[42] M. Kargarian, Entanglement properties of topological color codes, Phys. Rev. A78 (2008)062312, arXiv:0809.4276. 
[43] C. Vafa, Lectures on Strings and Dualities, arXiv:hep-th/970220.

[44] A. Belhaj, H. Ez-Zahraouy, M. B. Sedra, Toric Geometry and String Theory Descriptions of Qudit Systems, J. Geom. Phys. 95 (2015)21, arXiv:1408.3952.

[45] F. Holweck, J-G. Luque, M. Planat, Singularity of type $D_{4}$ arising from four qubit systems, arXiv:1312.0639.

[46] F. Holweck, H. Jaffali, Three-qutrit entanglement and simple singularities, arXiv:1606.05537.

[47] R.Kallosh, A.D.Linde, Strings, black holes, and quantum information,Phys.Rev. D 73 (2006) 104033 arXiv:hep-th/0602061.

[48] S.Ferrara, A.Marrani, Symmetric Spaces in Supergravity, Contemp. Math. 490(2009) 203, arXiv:0808.3567 [hep-th]. 